\title{
Configuration of High Performance Apartment Buildings Renovation: a constraint based approach
}

\author{
É. Vareilles ${ }^{1}$, A. F. Barco Santa ${ }^{2}$, M. Falcon ${ }^{1,3}$, M. Aldanondo ${ }^{1}$, P. Gaborit $^{1}$ \\ ${ }^{1}$ Mines Albi-Carmaux, University of Toulouse, Albi, France \\ ${ }^{2}$ Pontificia Universidad Javeriana - Cali, Santiago de Cali, Colombia \\ ${ }^{3}$ TBC Générateur d'Innovation, Colomiers, France \\ (corresponding author email: elise.vareilles@mines-albi.fr)
}

\begin{abstract}
This paper looks at the possibility of configuring high performance renovation of apartment buildings by the use of a constraint satisfaction problem (CSP). A new thermal envelope wraps the whole building and allows it to achieve a really low energy performance of $25 \mathrm{kWh} / \mathrm{m}^{2} /$ year. This new thermal envelope is composed of rectangular panels, always comprising insulation and cladding, and sometimes including, in addition, doors, windows or solar modules. The panels can be fixed directly onto the walls or onto a metal structure around the whole building. The decision support system, based on CSP approaches, will simultaneously enable the interactive definition of the renovation, the associated bill of material (BOM) and the building site assembly process. The range of knowledge to exploit and to model leads us to integrate into a single decision support system different constraint types, such as classical, dynamic, geometric and global constraints, as well as their filtering methods.
\end{abstract}

Keywords - BOM, Buildings Renovation, Configuration, Constraint Satisfaction Problem, Decision Support System.

\section{INTRODUCTION}

The global contribution from buildings (residential and commercial) towards energy consumption has steadily increased. Buildings account for around $20 \%$ and $40 \%$ of the total final energy consumption in developed countries: $37 \%$ in the EU [1], 36\% in the USA [2] and $31 \%$ in Japan [3]. Nowadays, it has exceeded industry and transportation sectors. Growth in population, enhancement of building services and comfort levels, together with the rise in time spent inside buildings assure the upward trend in energy demand will continue in the foreseeable future. Reducing energy consumption of the building sector is therefore one of our century challenges.

In several countries, research works are carried out on the efficient measures to take in order to reduce energy consumption of the building stock. Most states set regulations to improve the energy performance of new buildings. However, the annual rate of construction of new dwellings is only $1.1 \%$ in Europe [4]. It is therefore very important to renovate the existing buildings to reduce their energy consumption and to assist the retrofit process by the development of relevant decision support systems [5].

This study is one part of a research project called $C R I B A$, which aims to industrialize high performance thermal renovation of apartment buildings. In this project, a very innovative renovation system based on large timber frame panels will be designed. Moreover, several tools needed to industrialize the renovation process will be developed: a) a new method for three-dimensional building survey and modeling, b) a configuration system for the design of the building new thermal envelope (bill of material and assembly process), and c) a working site planning model with resource constraints.

The aim of this paper is to present a prospective study on the development of the interactive configuration system for the renovation of apartment buildings. Therefore, the paper is organized as follows. In section 2, we present the building renovation problem and how the configuration system can support apartment buildings renovation. In section 3 , we characterize the main configurable components of the renovation bill of material. In section 4, we sketch out the building renovation configuration process. In section 5 , we put forward some ideas on the different kinds of constraints that seem needed in order to make the apartment building renovation model.

\section{BUILDING RENOVATION CONFIGURATION NEEDS}

In this section, we introduce the building renovation problem, which is at the origin of our work. Then, we set out what the configuration system is expected to provide as results.

Building Renovation Problem. The industrialized high performance thermal renovation is based on an external new thermal envelope, which wraps the whole buildings. This new thermal envelope is composed of rectangular panels. Although the shape of the panels is a major limitation for the architectural creativity, this assumption is the key of renovation industrialization and matches most of apartment buildings that we target. Those panels always comprise insulation and cladding, and sometimes include in addition, doors, windows and solar modules.

The building sector is very dependent on hand-made methods, which are not always synonymous with quality guarantee [6]. Therefore, the aim of the CRIBA project is to prefabricate all the panels needed for a renovation. The panels are produced, delivered to the working site and finally hung on the facades in their producing order. Therefore, the renovation process enables thermal renovations: 
- at low cost, considering all the positive elements, fixed cost, logistic, etc,

- in a short time,

- of high quality,

- in a good environmental balance,

- without rehousing the inhabitants during the renovation works.

Depending on the building strength of materials, the panels can be fixed directly onto the facades or onto a metal skeleton around the whole building. With the new thermal envelope and equipment, the building must achieve a really low energy performance of 25 $\mathrm{kWh} / \mathrm{m}^{2} /$ year. In order to reach such a low energy performance, the new thermal envelope has to wrap perfectly the whole building. All the facades are covered by non- overlapping panels and are space-partitioned.

Building Renovation Configuration. The interactive configuration system for the renovation of apartment buildings will simultaneously enable the interactive definition of the renovation thanks to the associated bill of material and the building site assembly process.

The bill of material is a list of the components and sub-components, sub-assemblies, and the quantities of each needed to manufacture an end product. It can have multiple options and variants [7]. In our case, we consider:

- the new thermal envelope as the end product;

- the facade new envelopes as the sub-assemblies;

- the complete panels as sub-components.

The configurable components as leaves of the bill of material $(\mathrm{BOM})$ are therefore:

- the panels, which are placed on the facades, include wood structure, insulation and cladding (three or four different types),

- the angles, which make the junction between two facades. An angle is a specific type of panel which cannot include other components,

- the windows, doors, solar modules and balconies,

- the metal fasteners, which are used to fix either metal profiles or directly the panels onto the facades. There are several types of metal fastener depending on their type (fasteners to fix metal profiles, to hang panels or to provide wind bracing of panels), their load bearing capacity and the distance between the structural elements of the present facade and the panels,

- the metal profiles, which are used when the structural elements of the present facade cannot support the load of the new envelope. They are fixed onto the metal fasteners and the panels are hung on them. There is only one type of metal profile but its length has to fit the facade height.

The assembly process consists in a set of tasks to be carried out in order to assemble the new frame and envelope all around the building. It comprises some tasks that have always to be carried out, such as positioning and fixing metal fasteners and some that are optional, such as fixing the metal profiles onto the metal fasteners.

At least, the configuration system will give an idea of the renovation global cost, which includes the costs of raw materials, transportation, labor and lifting devices.

On the first hand, in the configuration community, many authors (among them [8], [9]) have shown that product configuration could be efficiently modeled and aided when considered as a Constraints Satisfaction Problem (CSP) [10]. On the other hand, in the civil engineering community and in the constraints community, many authors ([11], [12], [13], [14] or [15]) have shown that spatial layout can be solved with CSP. Consequently, we address the building renovation configuration with constraints and filtering algorithms.

\section{GENERIC BOM MODEL}

In this paper, we focus on the interactive definition of the renovation bill of material. In this subsection, we highlight the main variables of two configurable components: the panels and the angles. At the end of the configuration, all the configurable components variables have a single value, either given by the user or deduced by the configuration system.

Panels. The panels are rigid 2D rectilinear rectangles. Therefore, their sides are parallel to the facade reference axis. Let us consider one facade. All the panels covering it belong to a unique vertical plane. They are adjacent (they are at least one side in common) and are not overlapping themselves. By the way, they have all the same orientation. They cover completely the facade and make a partition of it.

The main variables of a panel refer to:

- its width $\left[\min _{\mathrm{w}}, \max _{\mathrm{w}}\right]$,

- its length $\left[\min _{1}, \max _{1}\right]$,

- its coordinates (abscissa and ordinate),

- its insulation thickness $\left[\min _{\mathrm{i}}, \max _{\mathrm{i}}\right.$ ],

- the insulation type (mineral wool or cellulose),

- its weight, which depends on its dimensions, the insulation type, and the components that are included in itself.

If the panel includes other components (windows, doors or solar modules), we need to know exactly for each of them:

- its width $\left[\min _{\mathrm{w}}, \max _{\mathrm{w}}\right]$,

- its length $\left[\min _{1}, \max _{1}\right]$,

- its relative position on the panel ( $\mathrm{x}$ and $\mathrm{y}$ ),

- its type and reference code.

A minimal distance is required between the sides of the panel and the position of other components in order to preserve the panel stiffness. 
Angles. The angles are rigid 3D L-polyomino tetracubes, which are placed at the building corners. The corners are at the intersection of two consecutive and perpendicular facade planes. We assume that the angles are right and do not include other components. Otherwise, a specific design task must be carried out in order to design the relevant angles.

Let us considering a facade corner. All the angles covering it belong to a unique vertical row. They are adjacent (they are at least one side in common) and are not overlapping themselves. By the way, they have all the same orientation. They cover completely the corner and make a partition of it.

The main variables of an angle refer to:

- its width [ $\min _{\mathrm{w}}, \max _{\mathrm{w}}$ ],

- its right length $\left[\min _{\mathrm{rl}}, \max _{\mathrm{rl}}\right]$,

- its left length $\left[\min _{11}, \max _{11}\right]$,

- its coordinates (abscissa and ordinate),

- its insulation thickness $\left[\min _{\mathrm{i}}, \max _{\mathrm{i}}\right.$ ],

- the insulation type (mineral wool or cellulose),

- its weight, which depends on its dimensions and the insulation type.

The angles dimensions directly depend on the sizes of their adjacent panels, with a minimal length (right and left) in order to preserve their stiffness.

\section{BUILDING RENOVATION CONFIGURATION PROCESS}

The building renovation configuration is a top-down and a five-step process. The process progressively converges from the working site to the configurable components. By a questions and answers game, the user inputs information and data in order to configure the renovation. After each user input, the configuration system removes inconsistent values and guides progressively the user to a solution. The user has to follow this process and gives information on:

Step 1) Working site: the working site description has an impact on the panels' dimensions. Let us focus on the working site accessibility and its local atmosphere. Concerning its accessibility, if you can access the working site with special convoys, the panels can be as wide and long as needed. Otherwise, the dimensions of the panels are constrained by the size of the trucks, which can access to the working site. Concerning the local atmospheric, if the working site area is very windy, the panels have to be smaller in order to be fixed onto the facades, and the renovation lasts longer because of nonworking periods.

Step 2) Block of apartment buildings: the block description has directly an impact on the hoisting equipment and indirectly on the panels' dimensions. If the block cannot be accessible with a tower crane, the panels have to be smaller in order to be conveying to the facades with suitable hoisting equipment, such as a telescopic boom lift.

Step 3) Apartment building: the apartment building description has an impact on the panels' dimensions. Let us consider only the apartment building height. If the apartment building height is lower than twelve meters (four stories), the height of the panels can be the same as the building one so that the panels are fixed vertically on the facades.

Step 4) Facades. Let us focus on a facade.

- First of all, the user has to describe precisely the structure and the geometry of the facade. Considering the structure of the facade, the user has to know where the metal fasteners can be fixed on the facade. A structural study has to be carried out in order to characterize the load bearing capacity of every area of the facade. Considering the geometry, the positions of windows, doors and balconies have to be known precisely. Only a topographic survey can provide this information.

- Regarding these areas and their characteristics, the decision of fixing the panels directly on the facade or on the metal profiles can be made. This decision has an impact on the BOM (metal fastener type and optional metal profiles) and on the assembly process (tasks devoted to metal profiles, such as delivery, assembly and adjustment).

- Having information about the working site, the block, the apartment building and the facade, the drawing of the facade layout can start. The user has now to indicate what the aesthetic effect (s)he wants (for instance, continuous vertical joints or a checkerboard effect).

- Knowing the facade layout, each panel has to be configured. If the panel is towards the south, it can be decided to add solar modules. If the panel has to include other components, the suitable reference code has to be selected for each of them.

Step 5) Angles: the renovation configuration finishes by the configuration of the angles. At this step, only the height of angles has to be determined.

At any time in every step of the configuration process, the user can change her/his inputs and see their impact on the configuration solutions. We have to notice that some decisions have an impact on all the configuration BOM. For instance, the choice of the cladding can be made in the first step and has an impact on all the panels and angles. All the facades are covered by the same cladding. However, some general choices can be discussed locally at a facade or a panel scale. For instance, the type of windows is made a priori for the whole building. 
However, if a specific existing window is too narrow to be replaced by a new external one (glazing size too low), another decision (keeping the existing window) can be made locally.

\section{BUILDING RENOVATION CONFIGURATION AND CONSTRAINTS}

Interactive renovation configuration is provided by constraint propagation that prunes bad solutions and progressively guides the way to good ones. In buildings renovation, the range of knowledge to exploit leads us to integrate into a single decision system different constraints types as well as their filtering methods.

Classical CSP Approaches. In building renovation configuration, we have to formalize different kinds of knowledge relevant to:

- civil engineering regulations that must be follow absolutely to the letter. For instance, fire barriers have to be installed between two consecutive stories in order to stop the spread of fire,

- civil engineering know-how that is the core knowledge of the companies involved in the CRIBA project,

- working site assembly process that allows us to define the suitable way of assembling the new frame and envelope all around the building.

As the range of knowledge to model is wide, we need to use different CSP approaches and their filtering algorithms, such as discrete CSP ([10], [16], [17], [18]), continuous CSP ([19] or [20]) and mixed CSP ([21]) depending on the type of the variables (discrete, continuous, symbolic or numeric) and the type of constraints (compatibility constraints or mathematical formula).

Groups and multi-instances of Constraints. In the renovation configuration, we have to cope with several instances of the same configurable components. For instance, in order to cover a facade with its new envelope, we need to configure $\mathrm{x}$ times a panel (such as described in subsection 3.1). We do not know in advance how many panels will be necessary, as it depends on a lot of data (working site description, block description, etc.). Therefore, we need to group variables and constraints into sets or classes, which can be instantiated as much as needed.

Dynamic Constraints. We have seen in the building renovation configuration process, section 4 , that we can decide to fix the panels on a metal envelope, or to create new openings on a facade. These decisions imply firstly the consideration of new components in the BOM and secondly, the insert of their assembly tasks in the assembly process. Therefore, we need to take into account the activation of configurable components as defined by [22], [23] and [24].
Geometric Constraints. In order to prefabricate the panels, we need to determine precisely the dimensions and the position of each component on the panels. The accuracy of the topographic measures and the precision of the components dimensions and position are the crucial for the industrialization of the building renovation and the goals of the CRIBA project. Therefore, in order to do so, we need to integrate to the configuration system geometric constraints (for a complete survey, see, [25] or [26], and for more recent work see [27], [14] or [15]).

Global Constraints. As we cannot know in advance how many panels are needed to cover a facade, we have to cope with constraints that depend on the number of instances of the same class. For instance, if the height of the facade is covered with more than one panel, the sum of all the panels' heights has to be equal to the facade one. Therefore, we need to integrate and filter different kinds of global constraints [28].

\section{CONCLUSIONS}

The aim of this paper has been to present a prospective study on the development of the interactive configuration system for the buildings renovation.

The apartment buildings renovation configuration is a challenge however you look at it. First of all, we want to industrialize a process that is nowadays traditionally made by craftsmen. This point is quite a revolution for the civil engineering field where only few industrial engineering methods are applied and in particular in SMEs. Secondly, in order to be able to use a configuration system, we need to extract, validate and formalize relevant knowledge. In our application field, the nature and the origin of knowledge are quite various. We have therefore to use different types of variables and constraints. The filtering engine has therefore to integrate different kinds of propagation methods. Thirdly, we need to cope with different variables priorities. For instance, all the variables which describe the whole working site have a strong impact on the dimensions of the panels and cannot be changed. If an inconsistent solution is found, we could propose to the user to change her/his choices firstly on the panels and then to progressively zoom out to the whole working site.

As we are still in the very earliest stage of the CRIBA project and as apartment building renovation configuration is quite a complex process, we need to model in details the BOM components and their constraints. We have then to select, analyze, adapt and integrate constraints approaches and filtering algorithms in our propagation engine CoFiADe. CoFiADe has already been used for supporting heat treatments configuration [29], simultaneously product and planning configurations [30] and helicopters maintenance configuration [31]

\section{ACKNOWLEDGMENT}


The authors wish to acknowledge the TBC Générateur d'Innovation company, the Millet and SyBois companies and all partners in the CRIBA project, for their involvement in the construction of the CSP model.

\section{REFERENCES}

[1] L. Perez-Lombard, J. Ortiz, and C. Pout. A review on buildings energy consumption information. Energy and Buildings, 40(3):394 - 398, 2008.

[2] U.S. Green Building Council. New Construction Reference Guide, 2013.

[3] The Energy Conservation Center. Energy Conservation Handbook. Japan, 2012.

[4] B. Poel, G. van Cruchten, and C.A. Balaras. Energy performance assessment of existing dwellings. Energy and Buildings, 39(4):393-403, April 2007.

[5] Y.K. Juan, P. Gao, and J. Wang. A hybrid decision support system for sustainable office building renovation and energy performance improvement. Energy and Buildings, 42(3):290-297, March 2010.

[6] M. Falcon and F . Fontanili. Process modeling of industrialized thermal renovation of apartment buildings. In eWork and eBusiness in Architecture, Engineering and Construction, European Conference on Product and Process Modelling (ECPPM 2010), September 2010.

[7] A. Felfernig. Standardized configuration knowledge representations as technological foundation for mass customization. In IEEE Transactions on Engineering Management, volume 54, pages 41-56, February 2007.

[8] D. Sabin and R. Weigel. Product configuration frameworks a survey. In IEEE Intelligent Systems, volume 13, pages 4249, 1998.

[9] T. Soininen, T. Tiihonen, T. Mnnist, and R. Sulonen. Towards a general ontology of configuration. Artificial Intelligence for Engineering Design, Analysis and Manufacturing, 12(4):357-372, 1998.

[10] U. Montanari. Networks of constraints: fundamental properties and application to picture processing. In Information sciences, volume 7, pages 95-132, 1974.

[11] K. Honda and F. Mizoguchi. Constraint-based approach for automatic spatial layout planning. In Proceedings of the 11th Conference on Artificial Intelligence for Applications, CAIA '95, Washington, DC, USA, 1995. IEEE Computer Society.

[12] U. Junker. Handbook of Constraint Programming, chapter Chapter 24. Configuration. Elsevier, 2006.

[13] B. Medjdoub and B. Yannou. Dynamic space ordering at a topological level in space planning. In Artificial Intelligence in engineering, volume 15, pages 47-60, January 2001.

[14] M. Zawidzki, K. Tateyama, and I. Nishikawa. The constraints satisfaction problem approach in the design of an architectural functional layout. Engineering Optimization, 43(9):943-966, 2011.

[15] F. Regateiro, J. Bento, and J. Dias. Floor plan design using block algebra and constraint satisfaction. Advanced Engineering Informatics, 26(2):361-382, April 2012.

[16] A.K. Mackworth. Consistency in networks of relations. In Artificial Intelligence, volume 8(1), pages 99-118, 1977.

[17] C. Bessire and M.O. Cordier. Arc-consistency and arcconsistency again. In AAAI, pages 108-113, 1993.

[18] B. Faltings. Arc consistency for continuous variables. In Artificial Intelligence, volume 65, pages 363-376, 1994.
[19] O. Lhomme. Consistency techniques for numeric CSP. In International Joint Conference on Artificial Intelligence, pages 232-238, Chambry, France, Aot 1993.

[20] F. Benhamou, D. Mc Allester, and P. Van Hentenryck. Clp(intervals) revisited. In ILPS'94, pages 1-21, 1994.

[21] E. Gelle. On the generation of locally consistent solution spaces in mixed dynamic constraint problems. Thèse de doctorat, École Polytechnique Fédérale de Lausanne, Suisse, 1998.

[22] S. Mittal and B. Falkenhainer. Dynamic constraint satisfaction problems. In AAAI, pages 25-32, Boston, US, 1990.

[23] D. Sabin and E.C. Freuder. Configuration as composite constraint satisfaction. In Artificial Intelligence and Manufacturing Research Planning Workshop, pages 153161, 1996

[24] B. Faltings, D. Sam-Haroud, and I. Smith. Dynamic constraints propagation with continuous variables. European Conference on Artificial Intelligence, pages 754 758, 1992.

[25] Maurice Dohmen. A survey of constraint satisfaction techniques for geometric modeling. Computers \& Graphics, 19(6):831-845, 1995.

[26] I. Fudos and C. Hoffmann. A graph-constructive approach to solving systems of geometric constraints. ACM Transactions on Graphics, 16(2):179-216, 1997.

[27] C. Jermann, G. Trombettoni, B. Neveu, and M. Rueher. A constraint programming approach for solving rigid geometric systems. In Constraint Programming, Singapore, 2000.

[28] Willem-Jan van Hoeve and Irit Katriel. Handbook of Constraint Programming, chapter Chapter 6. Global Constraints. Elsevier, 2006.

[29] M. Aldanondo, K. Hadj-Hamou, and Paul Gaborit. A constraint based approach for aiding heat treatment operation design and distortion evaluation. In Artificial Intelligence Applications and Innovations, volume 204 of IFIP International Federation for Information Processing, pages 254-261. Springer US, 2006.

[30] E. Vareilles, M. Aldanondo, M. Djefel, and P. Gaborit. Coupling interactively product and project configuration: a proposal using constraints programming. In International Mass Customization and International Conference on Economic, Technical and Organisationel Aspects of Product Configuration Systems, June 2008.

[31] E. Vareilles, C. Beler, E. Villeneuve, M. Aldanondo, and L. Geneste. Interactive configuration and time estimation of civil helicopter maintenance. In Workshop on Configuration in the European International Joint Conferences on Artificial Intelligence, Los Angeles, California, USA, July 2009. 